\title{
Note de recherche La protection du marché du soja en Indonésie.
}

\section{Justifications et contraintes}

\section{Frédéric Lançon}

\section{Citer ce document / Cite this document :}

Lançon Frédéric. Note de recherche La protection du marché du soja en Indonésie. Justifications et contraintes. In: Économie rurale. N²34-235, 1996. Globalisation des économies agricoles et alimentaires. Situation et prospective. p. 45;

doi : https://doi.org/10.3406/ecoru.1996.4804

https://www.persee.fr/doc/ecoru_0013-0559_1996_num_234_1_4804

Fichier pdf généré le 08/05/2018 


\section{La protection du marché du soja en Indonésie Justifications et contraintes}

e démantèlement des barrières douanières engagé _depuis la fin des années quatre-vingt touche de façon inégale les principaux produits vivriers. A ce jour, seules les importations de maïs ont été totalement libéralisées. S'il y a un consensus pour le maintien de la protection du marché du riz, sur lequel repose la politique alimentaire, qui a largement contribué à la stabilité politique du pays, en revanche, le contrôle des importations de soja est l'objet d'une controverse entre les décideurs politiques. La vivacité de ce débat est due au statut particulier du soja. Il est à la fois un produit important du système alimentaire traditionnel en fonction duquel s'est développée tagriculture indonésienne mais il est aussi un produit (I'alimentation animale en Jileine croissance. Le soja est donc au cœur de la m.station du secteur agro-alimentaire indonésien ir.duite par les changements quantitatifs et qualitatifs 'E la demande.

1 e contingentement des importations est un élément L du programme de relance de la production mis en place au début des années quatre-vingt pour répondre á la forte croissance de la consommation d'aliments à base de soja, dont la fabrication utilise à cette époque plus de $90 \%$ des disponibilités. Le but de cette mesure, soutenir les prix au producteur, est atteint parce qu'il n'y a pas de matières premières agricoles substituables au niveau de la transformation, ni de sources d'approvisionnement alternatives (importations) pour ces produits frais au niveau du consommateur. Le degré de concurrence élevé, qui règne en aval de la filière entre de multiples intervenants, les empêche de s'accaparer cette rente qui est donc largement redistribuée au producteur. Enfin, la croissance des revenus et la faible part des dépenses alimentaires consacrées à l'achat de ces produits limitent les effets négatifs de cette hausse des prix sur la consommation qui continue à se développer. Cette hausse des prix au producteur est un facteur capital dans le développement rapide de la production qui double en dix ans (14000 000 tonnes en 1994).

Lars a filière tourteau liée à l'expansion très rapide de la production industrielle de viande se développe dans un contexte beaucoup plus concurrentiel. Ce produit est l'objet d'un commerce international important et peut être partiellement remplacé par d'autres sources de protéines dans les formulations. L'intervention du gouvernement dans cette filière est beaucoup plus contraignante. En 1988, une unité de trituration démarre en bénéficiant d'accord préférentiel avec le gouvernement. Alors que jusqu'en 1988 la totalité du tourteau est importée, le gouvernement décide d'interdire les importations pour faciliter l'écoulement de la production de l'usine. La forte hausse des prix induite par ce monopole oblige les fabricants d'aliments pour bétail à réduire au minimum la part du tourteau dans leurs formules, remettant en cause les performances des aliments produits. Ceci devient plus contraignant à partir de 1990 lorsque, suite à la libéralisation de la législation, des firmes internationales, ayant la maîtrise des différents stades de la filière, investissent dans des élevages de grandes capacités nécessitant des aliments de bonne qualité.

A partir de 1991, sous la pression croissante de ces industriels, les importations de tourteaux reprennent et sont totalement libéralisées quatre ans plus tard. Par contre, les importations de graines restent contrôlées. Cette discrimination est révélatrice de la nature différente des enjeux propres à chacune de ces deux filières, de l'évolution du rôle des pouvoirs publics et des orientations politiques affichées. Le changement de position dans le cas du tourteau montre qu'avec l'arrivée des investisseurs étrangers, les relations entre le gouvernement et l'industrie ne peuvent plus être régies dans le seul cadre d'alliances avec des conglomérats nationaux mais que l'État doit aussi devenir un arbitre. Vis-à-vis des partenaires commerciaux, cette libéralisation des importations est aussi un gage de l'engagement vers une ouverture croissante des frontières. Le maintien de la protection du marché du soja-graine témoigne de la volonté des pouvoirs publics de minimiser les écarts croissants entre le revenu des agriculteurs et celui des autres catégories de la population active. Cette prise en considération du coût social de la transformation de l'agriculture, induite par l'ouverture croissante de l'économie indonésienne, est indispensable. En effet, elle contribue à maintenir un climat social et politique stable sans lequel le processus d'ouverture et de globalisation ne saurait être durable.

\section{Frédéric LANÇON \\ CIRAD-CA}

